\title{
Type 1 dialbetic camp: An experience in Mauritius
}

Pravesh Kumar Guness ${ }^{1}$, T1Diams ${ }^{2}$

${ }^{1}$ BSc (Hons), MBChB, ${ }^{2}$ T1Diams, Nongovernment Organisation, Vacoas, Mauritius

Abstract The aim of the nongovernmental organization Type 1 diabetes mellitus support (T1Diams) is to empower children, adolescents, and young adults with Type 1 diabetes to optimally manage their medical condition. In the Republic of Mauritius (an Island in the South-West of the Indian Ocean), since 2007, T1Diams has been organizing a yearly diabetic camp of 7-day duration during the winter season for its members and their families. Two age groups are present ( $0-11$ years and $12-25$ years). For the first group, the children are accompanied by their parents while the second group comes on their own. During the camp, therapeutic education is carried out. The acquisition of knowledge and self-care skills are ensured through pedagogical diabetes-related games and presentations. Physical activities as well as workshop on carbohydrate counting are also organized. Those activities help the participants acquire and maintain the skills that are sine qua non to live optimally with their disease. During the camp, there are also interventions from professionals (nutritionist, psychologist, social worker, endocrinologist, ophthalmologist, podiatrist, and dentist) so that they get a complete medicosocial follow-up. Self-monitoring of blood glucose and education on insulin injections are done on a regular basis (at least 4 times/day). Tests for glycated hemoglobin and microalbuminuria are performed with all the patients.

Keywords: Diabetic camp, Mauritius, nongovernment organization, self-care, therapeutic education, Type 1 diabetes, Type 1 diabetes mellitus support

Address for correspondence: Dr. Pravesh Kumar Guness, Cite Intenationale, Chambre 618, 20 Rue Hippolyte Foucque, 97490 St. Clothilde,

Reunion, Paris, France.

E-mail: praveshkumarguness@gmail.com

Received: 11.10.2016, Accepted: 28.01.2017

\section{INTRODUCTION}

Every Mauritian diabetic camp ends with a strong feeling of satisfaction and all the staff (administration, logistics, medical team, and animators) and especially the patients with Type 1 diabetes eagerly await for the next camp.

The first diabetic camp was set up in Michigan in 1925, by Leonard FC Wendt. ${ }^{[1]}$ Nearly 90 years later, there are more than 360 diabetic camps throughout the rest of the world $^{[2,3]}$ and about 15,000-20,000 campers with Type 1 diabetes attend those diabetic camps. ${ }^{[1]}$

\begin{tabular}{|l|l|}
\hline \multicolumn{2}{|c|}{ Access this article online } \\
\hline Quick Response Code: & Website: \\
\hline & www.joshd.net \\
\cline { 2 - 2 } & DOI: \\
& 10.4103/joshd.J_Soc_Health_ \\
\hline
\end{tabular}

\section{TYPE 1 DIABETES MELLITUS SUPPORT AND TYPE 1 DIABETES}

In Mauritius Island, the prevalence of Type 2 diabetes in the age group 20-79 for the year 2014 is $23.5 \% \cdot{ }^{[4]}$ Only one study has been done on the epidemiology of Type 1 diabetes in Mauritius. ${ }^{[5]}$ There are about 500 patients with Type 1 diabetes diagnosed. The age-standardized average incidence of Type 1 diabetes is 4.94/100,000/year. ${ }^{[5]}$ Type 1 diabetes mellitus support (T1Diams), a Mauritian nonprofit organization, specialized in the care and self-management of Type 1 diabetes in Mauritius was founded in 2005. It

This is an open access article distributed under the terms of the Creative Commons Attribution-NonCommercial-ShareAlike 3.0 License, which allows others to remix, tweak, and build upon the work non-commercially, as long as the author is credited and the new creations are licensed under the identical terms.

For reprints contact: reprints@medknow.com

How to cite this article: Guness PK, T1Diams. Type 1 diabetic camp: An experience in Mauritius. J Soc Health Diabetes 2017;5:66-70. 
has been working on the development and implementation of diabetic therapeutic education since the past 10 years. It achieves its goals using diabetes-related games during home visits of members, organizing regularly diabetic recreation days, and holding an annual winter diabetic camp. The yearly diabetic camp is an opportunity to strengthen the knowledge of diabetes among their members. It has been successfully organizing this event for the past 8 years.

\section{THE IMPORTANCE OF A CAMP IN MAURITIUS}

The aim is to allow the patients to enjoy as well as to improve their knowledge and competence on the management of their medical condition in a nontraditional environment. It is an opportunity for them to be "separated" from their parents and to nurture an improved sense of community and friendship with people of the same age group and medical condition. It is also an occasion to perform clinical research ${ }^{[2]}$ that will enable T1Diams to promote the impact of diabetic camp internationally. The patients learn to live a normal lifestyle with their diabetic condition among other children with Type 1 diabetes. Furthermore, T1Diams' diabetic camp is a real world diabetic experience for nondiabetic volunteers to step in the shoes of patients with Type 1 diabetes. Finally, this diabetic camp is a unique opportunity to reinforce the experience of staff working in the association and to help the patients to have the best possible glycemic control. ${ }^{[2]}$

\section{Duration and funding}

T1Diams is the only expert nongovernmental organization with know-how in setting up diabetic camps in Mauritius. The diabetes management protocol is not significantly different from that used by the American Diabetes Association, ${ }^{[6]}$ Canada and the French Aide aux Jeunes Diabetiques (AJD). ${ }^{[7]}$ The specificity of these camps is that they are organized in winter with an average temperature of $14^{\circ} \mathrm{C}-16^{\circ} \mathrm{C}$. Winter season lasts from May to September. The T1 members are on school holidays for about 2 weeks, and the camp lasts for one whole week. About forty patients with Type 1 diabetes attend this camp annually. The camp's budget is funded by donations from the corporate sector; no funding from the government is available. In France, the association AJD organizes camps ranging from 1 to 3 weeks, and these are sponsored by the government and private donors. ${ }^{[]}$

\section{Diabetes management}

According to the Diabetes Control and Complications Trial Research Group, the goal for glycemic control during diabetic camps is to prevent hypoglycemia as in these camps the stay is short and associated with increased energy expenditure through more frequent physical activities. ${ }^{[8]}$ T1Diams also follows the same principle. Two months before the camp, a camp questionnaire is sent to the members where they fill in their name, address, weight, height, past medical history, any allergy, particular food regimen, immunization record, ongoing illness, history of severe hypoglycemia, diabetes regimen (types of insulin, dose and time of injection, and latest glycated hemoglobin [HbA1c]), and any psychosocial issues. These data are compiled and analyzed by a committee (consisting of diabetic educators, doctor, psychologist, and camp director) 1 week before the camp. On the camp, each member has a personal file where these data are available. They are advised to bring their glycemia logbook where any particular adjustment to the dose of insulin can be done when the latter returns home.

On this camp, every member has her/his own glucometer and does a test before each meal, at bedtime and in case of hypoglycemia or hyperglycemia. The values are recorded in the logbook and also on a large white board. The activities and meals are also recorded. According to one of the protocols used, hyperglycemia is corrected with a bolus of long-acting insulin, and $2 \mathrm{~h}$ after the injection, the glucose levels are checked. This is applied over a $24 \mathrm{~h}$ period. Any member attending the camp for the first time is encouraged to always check her/his blood glucose levels to check for hypo/hyperglycemia. These children benefit from the support of the elder patients with Type 1 diabetes.

For the reference blood glucose values, the recommendations from IDF/ISPAD guideline ${ }^{[9]}$ are used, i.e., 4.4-6.6 mmol/L fasting blood glucose, before lunch and before dinner. At bedtime, up to $10 \mathrm{mmol} / \mathrm{L}$ is considered acceptable.

\section{Age group and diabetic education}

The age range of members with Type 1 diabetes in T1Diams varies from 12 months to 18 years with an average of 14 years. The camp duration is 7 days. The age group is divided into two: $0-11$ years (children and parents) and 12 years and over (adolescents only). For the past several years, the first 4 days of the camp are devoted for adolescents only, whereas the remaining days are for the children and parents group. The objectives for the two groups are different. T1Diams follows the same recommendation as AJD, ${ }^{[7]}$ France, where they have different targets to achieve for different age groups.

- 0-11 years: These parents and children are new to Type 1 diabetes. It is an opportunity for them to learn the basics of Type 1 diabetes outside a hospital setting. They learn how to do a blood glucose test, know their equipment (glucometer, glucose strips, insulins, glucagon, and needles), learn how to plan a healthy diet, recognize the symptoms of hypoglycemia, and learn 
what Type 1 diabetes is. The parents are empowered on how to treat hypoglycemia and how to set up a hypokit for their children at school. Furthermore, it is an occasion where the children learn to perform a blood glucose test alone so that they are empowered to do it on their own at school. These children are educated through games and the numbers showing hypo-hyperglycemia

- Age 12 and above: The aim is to consolidate their skills on Type 1 diabetes management. On the camp, they will apply all the therapeutic education carried out during the year. For example, they learn how to adapt their doses of insulin or where to inject insulin in case of physical activities.

\section{Diabetes therapeutic education}

The diabetic camp is built on five pillars, namely:

- Diabetic education

- Technical plateau

- Sports

- Nutrition

- Peer group experience.

Diabetic education is done every day, on an individual basis or in groups. Games and educational tools are used to achieve the objectives.

The technical plateau includes strengthening the capacity of the children to perform a blood glucose test, carry out insulin injection, fill in the diabetic logbook, and learn how to recognize hypo-hyperglycemia and the respective treatment.

Sports include low intensity to vigorous physical activities. The aim is to create awareness on the importance of physical activities and to empower them to link Type 1 diabetes with sports and to remove the taboo that they cannot perform physical activities just because they are patients with Type 1 diabetes.

Nutrition plays a very important role on the camp. The meal plan is designed by a dietician to meet the energy and carbohydrate needs of patients with Type 1 diabetes s. The meals are also served as a teaching tool to better understand the concept of carbohydrate counting.

Further, the peer group experience enables the children of the same group age to meet and share their experience with each another on the management of their condition.

\section{Diabetic education}

On the camp, several educational topics on Type 1 diabetes are covered in view of empowering the members to manage their diabetes efficiently. The topics include:
- Human body function

- What is Type 1 diabetes

- Hypoglycemia

- Hyperglycemia

- Types of insulin

- Adaptation of insulin doses

- Nutrition

- Psychosocial issues

- Diabetes and pregnancy

- Diabetes complications

- Sports

- Insulin injection techniques

- Blood glucose monitoring

- Importance of HbA1c and microalbumin tests.

Medical team and staff training

The diabetic camp is a medical recreation camp comprising a doctor, a nurse, medical students, and a diabetes educator. A doctor experienced in the management of Type 1 diabetes is present. He instructs the nurses when there are medical emergencies and provides guidance and his expertise on any particular medical issue arising. He also makes the lists of drugs that are necessary on the camps for conditions such as anaphylactic shock, asthma, nonhypoglycemic fits, pain, fever, abdominal pain, vomiting, sore throats, and allergies. We also have a first aid kit for minor injuries. With the assistance of the administrative staff, they make provision for glucose strips, glucometers, insulin, and needles.

The nursing staff consists of one nurse and one diabetes educator who are assisted by medical students. Whenever there is an incident of severe hypoglycemia, the nurses are trained to put an intravenous line with glucose $30 \%$ or glucagon injection. The Ministry of Health of Mauritius regularly sends one or two diabetes nurses to attend and gain experience. The medical students are recruited from the University of Mauritius on as volunteers. One month before the camp, they are trained during a 2-day session on the management of Type 1 diabetes. Then, on the camp, they will do appropriate adjustment to insulin doses with respect to physical activities and food intake. All the time they will be under the supervision of the camp doctor. Furthermore, the nursing staff gets a crash course on Type 1 diabetes. During the training of the medical staff, each medical staff member is allocated her specific job description. ${ }^{[10]}$

Every day, the dietician is present on the camp. She plans the menu according to activities so that members do not fall in hypoglycemia during activities and could follow the same example at home or during outing. She designs menus that are acceptable to all cultural groups. 
During the camp, there are also interventions from professionals (nutritionist, psychologist, social worker, endocrinologist, ophthalmologist, podiatrist, and dentist) so that residents get a complete medico-social follow-up.

\section{Administration}

The camp is managed by an administrative staff. It consists of a manager, an administrative coordinator, and a field worker. The manager has the responsibility to ensure the overall smooth running of the camp. Meetings are scheduled on a daily basis to ensure that all activities and meals are properly planned for the following day. The smooth running of the daily camp routine creates the proper environment for members onsite to learn more about their diabetic condition that will help them when they return back home.

\section{Diabetes-related emergencies}

The medical staff is trained by a medical doctor on how to recognize and manage hypo/hyperglycemia.

\section{Hypoglycemia}

A protocol for hypoglycemia treatment is available in the medical room. Hypoglycemia is defined as a blood glucose level $\leq 3.3 \mathrm{mmol} / \mathrm{L}(0.6 \mathrm{~g} / \mathrm{L})$. Five grams of oral glucose per $20 \mathrm{~kg}$ of body weight is given, and we wait for $10 \mathrm{~min}$ for symptoms to subside. In case, there is persistence of hypoglycemia, we repeat the oral glucose dose. In case of severe hypoglycemia, the medical staff is trained on the administration of $30 \%$ glucose intravenous solution or intramuscular injection of glucagon. At $22.00 \mathrm{~h}$, a blood glucose value of $8-10 \mathrm{mmol} / \mathrm{L}$ is considered acceptable.

Before physical activity, the dose of insulin is decreased, and a $10 \mathrm{~g}$ glucose equivalent snack is given.

\section{Hyperglycemia}

In case of blood glucose readings $\geq 14 \mathrm{mmol} / \mathrm{L}$, the child is requested to do a urine ketone level test. There is a set protocol for the management of hyperglycemia with or without ketosis. For hyperglycemia without ketosis, the amount of insulin needed is calculated to correct the elevated blood glucose reading ( 1 unit of rapid-acting insulin $=99 /$ total dose of insulin over $24 \mathrm{~h}]$ ). In the presence of ketosis, the child is given water to prevent dehydration, and a calculated amount of insulin is given to reverse ketosis. No physical activity is allowed during that period. The child's condition is followed up using a flow sheet. If the symptoms persist, the child is referred to the nearby regional hospital.

\section{Glycated hemoglobin and microalbuminuria}

T1Diams is the only nongovernmental organization on the Island to have an HbA1c and microalbuminuria apparatus validated by the Government Central Laboratory of Mauritius. This point of care is of extreme importance for our members on the camp. The HbA1c test is done on all patients on the camp, and it serves as a reference because many patients do not even know their last values.

Since the patients report that they never did a urine test for microalbumin at the hospital, the test is done on the camp. The screening for microalbumin in urine is done for those living with more than 10 years of diabetes and who never had a microalbumin test done at the hospital. The test is done to detect diabetes-related kidney complications. If a child shows a positive test, a medical report will be provided to the treating doctor of the patient for prompt action.

\section{DIABETIC CAIMIP PROTOCOLS}

On the T1Diams' camp, there is a well-defined medical management protocol. This has been created by the staff of the association and medical professionals. It follows the American Diabetes Association's standards of medical care. ${ }^{[6]}$

The protocols available on the camp are:

- Diabetes management adapted from ISPAD ${ }^{[0]}$ and $\mathrm{AJD}^{[7]}$

- Hypoglycemia and its treatment

- Treatment for hyperglycemia with ketones

- Referral form to hospital

- Checklist for drugs, food, beverages, and waste disposals

- Incident and accident reporting.

\section{CONCLUSION}

During the past 8 years, T1Diams winter diabetic camp has positioned itself as a center of excellence for children with Type 1 diabetes to acquire skills on the management of their medical condition. In addition, they also gain from diabetic education, sports activities, healthy nutrition, and friendship from peer group experience. Being surrounded by well-trained professionals, a high standard of diabetes care is provided for the camp to function properly. T1Diams has been organizing these yearly residential camps for the past 8 years, thanks to its generous donors, and will continue to do so as its flagship activity.

Financial support and sponsorship

Nil. 


\section{Conflicts of interest}

There are no conflicts of interest.

\section{REFERENCES}

1. Kaufman F, Schatz D, Silverstein J; American Diabetes Association. Diabetes care at diabetes camps. Diabetes Care 2004;27 Suppl 1:S129-31.

2. Kaufman F, Schatz D, Silverstein J. Diabetes care at diabetes camps. Diabetes Care 2007;30:S74-6.

3. Diabetes Education and Camping Association. Diabetes Education and Camping Association; 2015. Available from: http://www. diabetescamps.org. [Last accessed on 2015 Apr 22].

4. IDF. Mauritius International Diabetes Federation; 2015. Available from: http://www.idf.org/membership/sea/mauritius. [Last accessed on 2015 Apr 22].

5. Guness P, Chan Sun M. Epidemiology of type 1 diabetes mellitus in mauritius. Am J Health Res 2013;1:32-5.

6. American Diabetes Association. Standards of Medical Care in Diabetes; 2016. Available from: http://www.care.diabetesjournals. org/content/27/suppl_1/s15.full.pdf. [Last accessed on 2015 Apr 22].

7. Available from: http://www.ajd-diabete.fr. [Last accessed on 2015 Apr 22]..

8. The effect of intensive treatment of diabetes on the development and progression of long-term complications in insulin-dependent diabetes mellitus. The Diabetes Control and Complications Trial Research Group. N Engl J Med 1993;329:977-86.

9. International Diabetes Federation. The Global IDF/ ISPAD Guideline for Diabetes in Childhood and Adolescence; 2014 Available from: http://www.idf.org/ global-idfispad-guideline-diabetes-childhood-and-adolescence. [Last accessed on 2015 Apr 06].

10. Skyler JS, editor. Medical Management of Type 1 Diabetes. $3^{\text {rd }}$ ed. Alexandria, VA: American Diabetes Association; 1998. 